\title{
Switchable static friction of piezoelectric composite-silicon wafer contacts
}

\author{
D. A. van den Ende, ${ }^{1,2,3, a)}$ H. R. Fischer, ${ }^{2}$ W. A. Groen, ${ }^{1,3}$ and S. van der Zwaag ${ }^{3}$ \\ ${ }^{1}$ Holst Centre-TNO, High Tech Campus 31, Eindhoven, The Netherlands \\ ${ }^{2}$ Materials for Integrated Products Department, TNO (Netherlands Organization for Applied Scientific \\ Research), Rondom 1, Eindhoven, The Netherlands \\ ${ }^{3}$ Novel Aerospace Materials group, Aerospace Engineering, Delft University of Technology, Kluyverweg 1, \\ Delft, The Netherlands
}

(Received 11 December 2012; accepted 26 March 2013; published online 10 April 2013)

\begin{abstract}
The meso-scale surface roughness of piezoelectric fiber composites can be manipulated by applying an electric field to a piezocomposite with a polished surface. In the absence of an applied voltage, the tips of the embedded piezoelectric ceramic fibers are below the surface of the piezocomposite and a silicon wafer counter surface rests solely on the matrix region of the piezocomposite surface. When actuated, the piezoelectric ceramic fibers protrude from the surface and the wafer rests solely on these protrusions. A threefold decrease in engineering static friction coefficient upon actuation of the piezocomposite was observed: from $\mu^{*}=1.65$ to $\mu^{*}=0.50$. These experimental results could be linked to the change in contact surface area and roughness using capillary adhesion theory, which relates the adhesive force to the number and size of the contacting asperities for the different surface states. C 2013 AIP Publishing LLC. [http://dx.doi.org/10.1063/1.4801459]
\end{abstract}

The manipulation of friction and adhesion of surfaces is interesting for many applications, such as precision manufacturing processes, where tolerances are becoming ever tighter and components are becoming smaller and more delicate. ${ }^{1}$ Also in micro-electro-mechanical systems (MEMS), friction and adhesion play a major role in lifetime issues due to smooth surfaces ${ }^{1-3}$ and low normal force conditions. ${ }^{4}$ Examples of engineered surfaces with controllable friction and adhesion include mechanical, ${ }^{5,6}$ thermal, ${ }^{7,8}$ and chemi$\mathrm{cal}^{9,10}$ control of the surface properties. Since friction is dependent on surface roughness, ${ }^{11-17}$ changing the surface roughness of flat surfaces can alter their frictional properties. Patterned piezoelectric materials will develop a non-uniform strain across their surface when actuated. ${ }^{18,19}$ These changes may change the macro-scale friction characteristics of the surface. Several concepts have been proposed that aim to achieve controllable friction through roughness modification. $^{20,21}$ Piezoelectric 1-3 or 2-2 (Ref. 22) composites can develop significant strain when actuated at high voltage. The relatively compliant polymer matrix is strained along with the active piezoelectric ceramic phase, although it is not piezoelectric itself. Due to this two phase, nature low volume fraction composites will develop a non-uniform strain profile across their surface ${ }^{19}$ changing the meso-scale surface roughness. In this study, the changes in static friction characteristics between piezoelectric composite and silicon wafer surfaces with applied voltage are measured. The friction change is correlated to the evolution of meso-scale roughness with voltage. Results indicate that low-volume fraction piezoelectric 1-3 composites display a distinct reduction in measured static friction against a smooth rigid counter surface at low normal force. Several advantages of using piezoelectric switchable friction surfaces over existing switchable friction surfaces ${ }^{5-10}$ are expected. These advantages include a fast switchability of the friction characteristics, as

a)daan.vandenende@tno.nl compared to for instance thermal ${ }^{8}$ or thermo-mechanical ${ }^{7}$ switching, a wide operating temperature range, as compared to thermally ${ }^{8}$ or chemically stimulated switching ${ }^{9}$ and an all solid state device, which is compatible with standard driving electronics.

The friction coefficient in the low normal force regime depends on the real contact area between two contacting solids, ${ }^{1,12}$ which is roughness dependent. ${ }^{23,24}$ Figure 1 depicts a schematic of the change in surface topology of a 1-3 composite. The piezoelectric phase, for instance lead zirconium titanate ceramic (PZT), deforms under the application of a voltage, while the polymer phase is passive and is only strained as a result of the PZT activity. If the PZT volume fraction is low, the deformation profile of the piezoelectric composite surface is non-uniform with peak strain occurring at the PZT locations. ${ }^{18,19}$

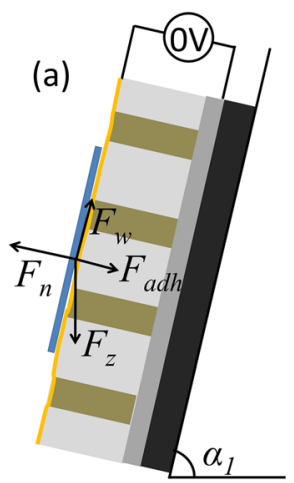

(b)

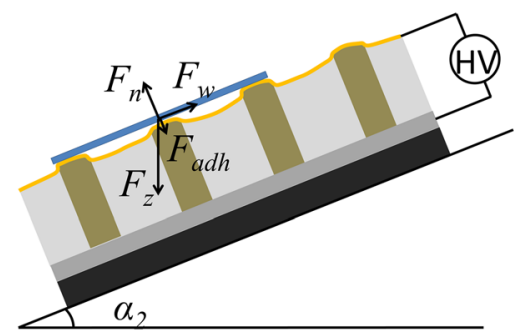

FIG. 1. Change of surface roughness of a 1-3 composite upon application of a voltage and demonstration of a sliding ramp measurement. When an electric field is applied, the PZT (dark grey) deforms actively, and the matrix (light grey) is only passively strained, causing an uneven strain profile at the surface. A counter-surface ( $\mathrm{Si}$ wafer, blue) is placed on the electrode piezocomposite surface (gold) and the piezocomposite device is positioned on a tilting substrate (black). (a) At $V_{a p p}=0 \mathrm{~V}$, the angle $\left(\alpha_{1}\right)$ at which the wafer starts sliding is high. (b) At $V_{a p p}>0 \mathrm{~V}$, the sliding angle $\left(\alpha_{2}\right)$ is reduced $\left(\alpha_{2}<\alpha_{1}\right)$. 
The static friction coefficient between two surfaces can be determined using a sliding ramp setup (see Figure 1). A counter-surface (silicon wafer) is placed on the surface of the composite, which is then tilted until static friction is overcome and the sample slides off the ramp. ${ }^{17}$ The apparent, or engineering-, friction coefficient, $\mu^{*}$, is determined using the externally applied normal force, $F_{n}^{e x t}$, and lateral friction force $F_{w}$ (see Eq. (1))

$$
\mu^{*}=\frac{F_{w}}{F_{n}^{e x t}} .
$$

In the sliding ramp experiment, $F_{n}^{e x t}$ is the component of the gravitational force, $F_{z}$ perpendicular to the contact plane, and the apparent friction coefficient is equal to the tangent of the sliding onset angle $\left(\mu^{*}=\tan \alpha\right)$.

When surfaces are smooth and low normal forces are applied, there is a risk that Eq. (1) will overestimate the friction coefficient. ${ }^{16}$ This is due to the contribution of the adhesion force, $F_{a d h}$, which is not taken into account in Eq. (1). ${ }^{17}$ This adhesion force contributes to the total normal force on the sample, $F_{n}^{\text {total }}$, and can become significant at low applied normal force. ${ }^{n}$ The real coefficient, $\mu$, equals

$$
\mu=\frac{F_{w}}{F_{n}^{\text {total }}}=\frac{F_{w}}{F_{n}^{e x t}+F_{a d h}} .
$$

The exact contribution of the adhesion force on the apparent friction coefficient cannot be determined from a single set of sliding ramp experiments. However, an indication on the switching ability of the piezocomposite surface can be obtained from the evolution of apparent friction behavior with applied voltage. The application of a voltage leads to a higher meso-scale surface roughness (see Figure 1) and therefore to a lower real contact area. Once the induced local displacements of the contact surface at the locations of the piezoelectric phase (i.e., the meso-scale surface roughness) increases beyond the interaction range of Van der Waals forces $^{3}$ and capillary forces ${ }^{13}$ the adhesion force decreases significantly. The decrease in adhesion force leads to a reduction in the apparent friction coefficient, $\mu^{*}{ }^{16}$

A set of 1-3 fiber composites were manufactured by embedding a $10 \times 10 \mathrm{~mm}^{2}$ array of PZT fibers (PZT5A fibers, Smart Material Gmbh, Germany) in epoxy. The array spacing was about $1 \mathrm{~mm}$. The fiber array was embedded in epoxy (Struers Epofix, Switzerland), and the PZT fibers were decoupled mechanically from the matrix epoxy by a thin low modulus coating. The samples were cut and electrically contacted to a rigid silicon backing using electrically conductive epoxy (CE3103, Henkel Germany). The samples were ground flat and to thickness (approximately $1 \mathrm{~mm}$ ) and subsequently polished. A $200 \mathrm{~nm}$ thick gold top electrode was sputtered on the surface directly. The surface on the PZT fibers and epoxy matrix regions are completely covered by the gold coating layer, ensuring that the surface contact during experiments is always silicon on gold. The top electrode was always electrically grounded during measurements. The edges of the wafer counter surface were ground at an angle with fine silicon carbide grinding paper (grit 4000) to avoid jagged edges, which can interfere with the measurement.
The wafer surface was cleaned by wiping with an acetone impregnated cloth, followed by cleaning in an ultrasonic bath in isopropanol and acetone for $5 \mathrm{~min}$ to remove particulates and blown dry with $\mathrm{N}_{2}$.

The surface of the (gold electroded) 1-3 PZT fiberepoxy composites was measured using a confocal microscope (Sensofar Plu2300). The surface roughness of the wafer counter-surface was measured on a white light interferometer (Sensofar Plu2300). Friction tests were performed on a sliding ramp setup (see Figure 1). At the start of the measurement, the silicon wafer counter surface was positioned slightly above the piezocomposite surface and dropped onto the surface. For the friction measurements, no extra pressure was exerted on the wafer after contact. The piezocomposite surface was actuated after contacting the wafer. The inclination of the sliding ramp was increased until the wafer released, indicating that the friction had been overcome. The sliding ramp motion was stopped and the angle was recorded at which the friction was overcome (average of 10 measurements). All experiments were executed at relative humidity of $23 \%$ and room temperature $\left(21^{\circ} \mathrm{C}\right)$ in a cleanroom environment in a down flow cabinet with local class 1000 environment.

An example of the switchable friction coefficient of the 1-3 piezocomposite surfaces is presented in the supplemental material. ${ }^{25}$ The wafer was held at $\alpha=90^{\circ}$ to prove sticking behavior by adhesion forces (i.e., $\mu^{*}=\infty$ ). After tilting the device back to $\alpha=60^{\circ}$, the voltage is switched on $\left(V_{\text {app }}=1500 \mathrm{~V}\right)$ and the wafer starts sliding instantly.

The measured apparent friction coefficient $\left(\mu^{*}=\tan \alpha\right)$ as a function of applied voltage is presented in Figure 2(a). The apparent friction coefficient drops significantly when an electric field is applied to the piezocomposite surface, which can be correlated to protrusion of the fiber asperities above the matrix surface. The evolution of surface height of one such fiber asperity with applied electric field is presented in Figure 2(b). The height difference between the center of the fiber and edges of the image area is plotted. Mechanical decoupling of the fiber from the matrix reduces the clamping effect of the matrix and maximizes the local protrusion of the surface. This effect is visible in the insets of Figure 2, where the surface of the matrix region shows no visible deformation upon application of a voltage, indicating effective decoupling. Further increase of the applied field does not result in changes in friction coefficient, since the fiber asperities are already above the matrix surface level. When a negative field below the coercive field of the PZT fiber is applied (i.e., PZT fibers contract), the friction coefficient returns to the zero field value, since the asperities are below the surface and, therefore, only the matrix surface is in contact with the silicon wafer (Figure 2(b)).

The surface of the 1-3 composites consists of regions around the embedded PZT fibers and free matrix area. The fiber roughness (Figure 3(a)) was measured to be $R_{\mathrm{RMS}}=45 \pm 8 \mathrm{~nm}$, with skewness, $\mathrm{R}_{\mathrm{sk}}=-5.7$ and kurtosis, $\mathrm{R}_{\mathrm{ku}}=60$. The large negative skewness and high kurtosis value is caused by the microstructure of the ceramic underneath the thin gold coating, which can be seen in Figure 3(a). Around the embedded PZT fibers, the polishing results in rounding of the edges of the fiber and matrix (see Figure 

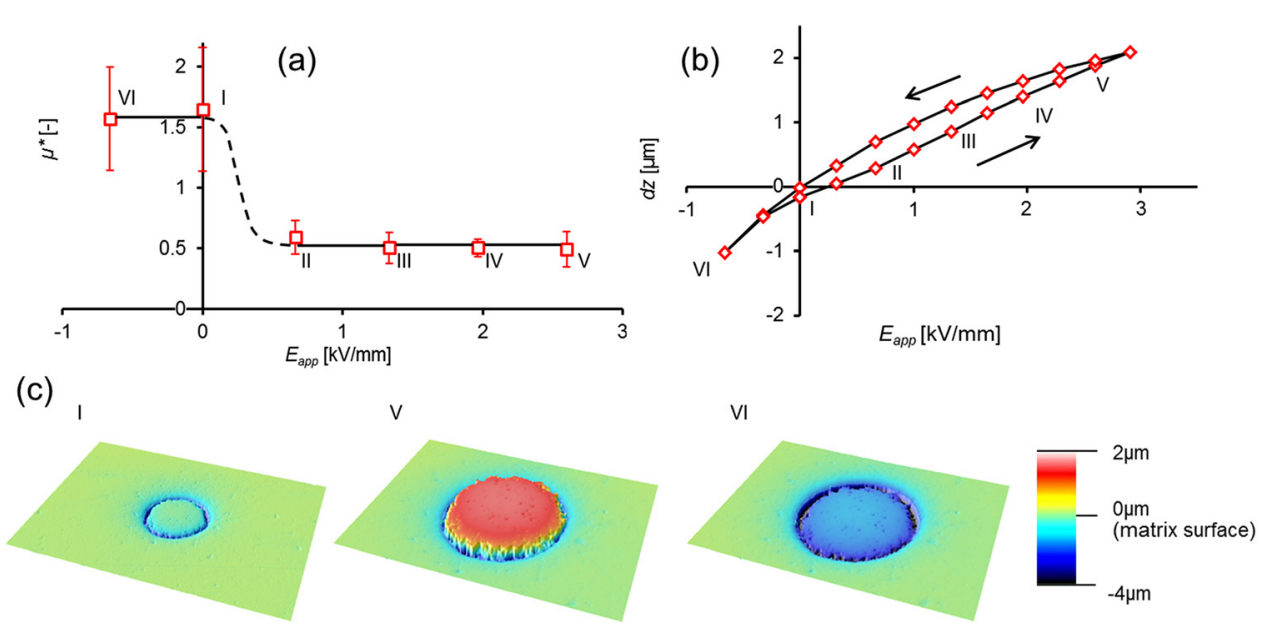

FIG. 2. (a) The apparent friction values, $\mu^{*}$, measured as a function of applied voltage (the measurement sequence is $E_{a p p}=0 \mathrm{kV} / \mathrm{mm}$ to $E_{a p p}=2.6 \mathrm{kV} / \mathrm{mm}$ followed by $E_{a p p}=-0.65 \mathrm{kV} / \mathrm{mm}$ ). (b) Evolution of asperity height as a function of voltage for a randomly selected PZT fiber asperity. The height difference $(d z)$ between the center of the fiber and edges of the image area is plotted (the measurement sequence is $E_{a p p}=-0.32 \mathrm{kV} / \mathrm{mm}$ to $E_{a p p}=2.9 \mathrm{kV} / \mathrm{mm}$ to $E_{a p p}=-0.65 \mathrm{kV} / \mathrm{mm}$ ). The data points corresponding to the measured friction values are marked I-VI. (c) The insets I, V, and VI represent confocal images of the asperity at different applied voltages (measured area inset I: $472 \times 340 \mu \mathrm{m}^{2}$, measured area insets V and VI: $254 \times 180 \mu \mathrm{m}^{2}$ ).

2(c)). Farther from the PZT fibers, the matrix surface is much flatter (see Figure 2(c) inset I). The surface roughness of the matrix region of the piezocomposite (Figure 3(b)) has a roughness of $R_{R M S}=4.8 \pm 1.4 \mathrm{~nm}$, with $R_{\text {sk }}=-0.44$ and $\mathrm{R}_{\mathrm{ku}}=6.0$. The measured surface roughness of the wafer counter-surface is $R_{R M S}=0.84 \pm 0.08 \mathrm{~nm}$.

The meso-scale roughness of the composites is best described by an array of asperities sitting just below the matrix surface, which can be actuated to protrude from the surface. Before actuation, the contacting surface consists of the gold electrode on epoxy. After actuation, the gold electrode on PZT contacts the counter-surface. The friction and adhesion force between two surfaces at low contact pressures depends on the contact area. ${ }^{2,23,26}$ For hard surfaces, such as gold covered epoxy and PZT, capillary forces from naturally existing water layers dominate the adhesion force between two smooth surfaces. ${ }^{14,27,28}$ Capillary condensation in small cavities between two closely contacting surfaces by wetting will result in the generation of attractive capillary forces. As a first order approximation, the capillary force, $F_{c a p}$, between a single asperity and a plate is considered. This force is a function of the asperity size and contact properties of the water with both surfaces. ${ }^{28}$ Depending on the layer thickness and surface roughness different wetting regimes occur. The toe-dipping regime regards a small layer thickness of the wetting fluid. This regime is generally valid at low relative humidity and yields an expression for $F_{c a p}$ of ${ }^{2}$

$$
F_{\text {cap }}=2 \pi \gamma_{l} r\left(\cos \theta_{1}+\cos \theta_{2}\right),
$$

(a) Gold coated PZT surface

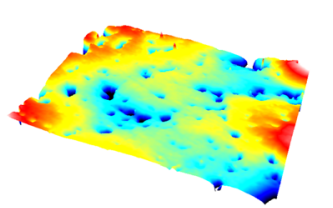

(b) Gold coated epoxy surface

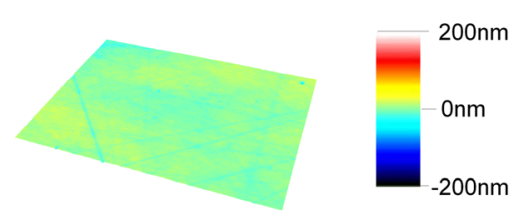

FIG. 3. Flattened confocal images of gold coated PZT ceramic surface (a) and gold coated epoxy surface (b). The measured area of each image is $85 \times 60 \mu \mathrm{m}^{2}$. where $\gamma_{1}$ is the surface tension of the wetting liquid, $r$ is the asperity radius of curvature, and $\theta_{1}$ and $\theta_{2}$ are the contact angles of the wetting liquid on both contacting surfaces. In the toe dipping regime, the liquid adhesion force of all wetted asperity contacts can be calculated by multiplying the single asperity force by the total number of contacts. ${ }^{2}$ The friction due to the external normal force, $F_{n}=-\cos \alpha F_{z}$, is independent of nominal contact area, according to Amontons friction law. ${ }^{23,28}$ However, the reduction in contact area does decrease the adhesive contribution to the normal force, $F_{\text {adh }}$, which influences the measured friction coefficient $\left(\mu^{*}=\tan \alpha\right)$. It has been shown that the adhesion force between two surfaces in ambient atmosphere is reduced by two orders of magnitude when the combined surface roughness increases from $1 \mathrm{~nm}$ to $10 \mathrm{~nm}$ for several different counter-surfaces on gold. ${ }^{13,29,30}$ The combined surface roughness of the matrix region of the piezocomposite and wafer is almost $6 \mathrm{~nm}$, which already yields relatively low adhesion forces between silicon and gold. ${ }^{13}$ After actuation, the wafer rests solely on the protruding PZT fibers. The PZT fiber surfaces have an appreciably higher roughness than the matrix surface, so the adhesive contribution per unit nominal area will be even lower still. ${ }^{13}$ Moreover, the contact to the PZT fibers results in a single asperity contact per fiber since the polishing results in a rounded summit as can be seen in the insets of Figure 2. Since maximum absolute height differences between individual fiber asperities in the array after polishing are about $500 \mathrm{~nm}$, it is safe to conclude that in total, only three asperities are in contact with the wafer once the piezocomposite is actuated, as dictated by static equilibrium requirements.

A first order estimation for the difference in adhesive contribution can be made by calculating the capillary contribution using Eq. (3), multiplied by the estimated number of asperities in contact. ${ }^{23}$ For this estimation, bulk water properties are used, $\gamma_{1}=72 \mathrm{~mJ} / \mathrm{m}^{2}$ and contact angles are $\theta_{1}=35^{\circ}$ ( $\mathrm{Si})$ and $\theta_{2}=70^{\circ}(\mathrm{Au})$. It has been shown that the capillary force calculation can be used for water layer thickness down to $2 \mathrm{~nm}^{2}$ Micro-roughness asperity parameters were 
estimated from the confocal images (see Figure 2(c)), $\mathrm{r}_{\text {epoxy }}$ $=0.05 \mathrm{~mm}$ and $\mathrm{r}_{\mathrm{PZT}}=0.01 \mathrm{~mm}$. Since the penetration depth is in the order of a few nanometers the elastic properties of only the gold electrode layer were used $(\mathrm{E}=70 \mathrm{GPa}$ and $\nu=0.42$ ). Using these properties average capillary forces of $F_{\text {cap }}=25 \mu \mathrm{N}$ per micro-asperity on the gold coated matrix surface and $F_{c a p}=5 \mu \mathrm{N}$ for a micro-asperity on the gold coated PZT fiber surface were estimated. In contrast, the Van der Waals force per asperity is less than $10 \%$ of the capillary adhesion force. Using the approximation for a sphere near a surface: $F_{v d W}=\frac{A_{132} r}{12 z_{0}}$, the force per asperity yields $F_{v d W}=0.4 \mu \mathrm{N}$ for gold coated PZT surface and $F_{v d W}=2 \mu \mathrm{N}$ for gold coated epoxy surface. For the Hamaker constant for silicon-gold contacts in the presence of water, $A_{132}$ $=20.35 \cdot 10^{-20} \mathrm{~J}$ (Ref. 31) was taken and a cut-off height of $z_{0}=0.6 \mathrm{~nm}$ was used. The dominance of the capillary adhesion force in this roughness regime is consistent with literature reports. ${ }^{14,27,28,30}$

For the actuated state, the first order approximation of the total adhesion force based on the capillary force per contacting asperity is $F_{a d h} \approx 16 \mu \mathrm{N}$, which is small compared to the normal force of the wafer $\left(F_{z} \approx 320 \mu \mathrm{N}\right.$ at $\left.0^{\circ}\right)$. Thus, the adhesive contribution to the friction coefficient is also small. At $\mu=0.5$, the difference between apparent friction coefficient $\mu^{*}$ (Eq. (1)) and the real friction coefficient $\mu$ (Eq. (2)) is around $5 \%$. In the non-actuated state, the wafer rests on gold-epoxy surface and the measured apparent friction coefficient is around $\mu^{*}=1.7$. If the real friction coefficient is around $\mu=0.5$, the extra adhesive force is around $475 \mu \mathrm{N}$, almost a 30 fold difference from the actuated case. Using the single asperity estimation of around $27 \mu \mathrm{N}$ adhesive force per contact for the gold on epoxy, still less than 20 asperities are contributing to the adhesion. Such a low number is not unreasonable, because the surface deformation depth is limited (around $1-3 \mathrm{~nm}$ ), since the normal force from the wafer weight and capillary adhesion is low. Moreover, the slight curvature of the surface $\left(\mathrm{r}_{\text {surface }} \approx 25 \mathrm{~m}\right.$ ) and rounded edges of the matrix close to the fiber were found, both due to polishing. This large scale non-flatness reduces the real contact area before actuation. The low number of contacting asperities also means that the measured friction is relatively sensitive to the precise number of contacting asperities, which may differ somewhat per measurement. This effect could explain the higher standard deviation of the measurement data in Figure 2(a) at $E_{\text {app }}=0 \mathrm{kV} / \mathrm{mm}$ and $E_{\text {app }}=-0.65 \mathrm{kV} / \mathrm{mm}$ compared to the measurements at $E_{\text {app }}>0$. The relatively low number of contacts before actuation also implies there is room for increasing the normal force contribution, by increasing the flatness and reducing roughness of the epoxy surface. This will ultimately increase the magnitude of the induced changes in the apparent friction coefficient.

In conclusion, the friction coefficient in the low normal force regime of 1-3 piezoelectric fiber-polymer composites against a silicon wafer can be manipulated by applying an electric field to the piezoelectric composite. In the low normal force regime, the measured apparent friction coefficient contains a significant contribution from the adhesion forces between the two surfaces. Actuation of the composite leads to a reduction in real contact surface area, which reduces the adhesive contribution to the normal force. Experimental results could be linked to the change in contact surface area and roughness using basic adhesion theory, which relates the adhesive force to the number and size of the contacting asperities. Controlling the friction of surfaces in this manner could be beneficial in precision mechanics devices, such as (miniaturized) positioning stages, where surfaces are often flat and hard, normal forces are often low and friction requirements for static and travelling states generally differ.

This work was financially supported by TNO and the Dutch government under the Smartmix funding Program (Grant SMVA0607), as part of the program "Smart systems based on integrated Piezo." The authors acknowledge Hans van der Veer (TNO) for his valued advice regarding sample preparation.

${ }^{1}$ A. Hariri, J. Zu, and R. Ben Mrad, J. Micromech. Microeng. 16, $1195-1206$ (2006).

${ }^{2}$ B. Bhushan, J. Vac. Sci. Technol. B 21, 2262 (2003).

${ }^{3}$ F. W. Delrio, M. P. De Boer, J. A. Knapp, E. D. Reedy, P. J. Clew, and M. L. Dunn, Nature Mater. 4, 629 (2005).

${ }^{4}$ J. Dunkin and D. Kim, Wear 193, 186 (1996).

${ }^{5}$ J. Lee, R. S. Fearing, and K. Komvopoulos, Appl. Phys. Lett. 93, 191910 (2008).

${ }^{6}$ L. F. Boesel, C. Greiner, E. Arzt, and A. del Campo, Adv. Mater. 22, 2125 (2010).

${ }^{7}$ S. Reddy, A. del Campo, and E. Arzt, Adv. Mater. 19, 3833 (2007).

${ }^{8}$ J. K. Chen, J. H. Wang, J. Y. Chang, and S. K. Fan, Appl. Phys. Lett. 101, 123701 (2012).

${ }^{9}$ L. Han, J. Yin, L. Wang, K. K. Chia, R. E. Cohen, M. F. Rubner, C. Ortiz, and M. C. Boyce, Soft Matter 8, 8642 (2012).

${ }^{10}$ M. C. LeMieux, Y.-H. Lin, P. D. Cuong, H.-S. Ahn, E. R. Zubarev, and V. V. Tsukruk, Adv. Funct. Mater. 15, 1529 (2005).

${ }^{11}$ C. Lee and A. Polycarpou, ASME J. Tribol. 129, 754 (2007).

${ }^{12}$ C. Yang and B. N. J. Persson, J. Phys.: Condens. Matter 20, 215214 (2008).

${ }^{13}$ P. J. Van Zwol, G. Palasantzas, and J. T. M. De Hosson, Appl. Phys. Lett. 91, 101905 (2007).

${ }^{14}$ P. J. Van Zwol, G. Palasantzas, and J. T. M. De Hosson, Phys. Rev. E 78, 031606 (2008).

${ }^{15}$ B. Bhusan, J. Israelaschvili, and U. Landman, Nature 374, 607 (1995).

${ }^{16} \mathrm{R}$. Heise and V. Popov, Tribol. Lett. 39, 247 (2010).

${ }^{17} \mathrm{H}$. Yoshizawa, Y. Chen, and J. Isrealachvili, J. Phys. Chem. 97, 4128-4140 (1993).

${ }^{18}$ W. Cao, Q. Zhang, and L. E. Cross, J. Appl. Phys. 72, 5814 (1992).

${ }^{19}$ Q. M. Zhang, W. Cao, and L. E. Cross, J. Appl. Phys. 73, 1403 (1993).

${ }^{20}$ A. L. Browne, N. L. Johnson, N. D. Mankane, N. K. Bucknor, G. P. McKnight, W. Barvosa-Carter, A. C. Keefe, C. P. Henry, and G. A. Herrera, US patent application US20090047197A1, 2009.

${ }^{21}$ E. C. Dillingh, C. G. M. Hermse, A. J. Huis in 't Veld, US patent application US20110081519A1, 2011.

${ }^{22}$ R. E. Newnham, D. P. Skinner, and L. E. Cross, Mater. Res. Bull. 13, 525 (1978).

${ }^{23}$ J. Greenwood and J. Williamson, Proc. R. Soc. London A 295, 300 (1966).

${ }^{24}$ P. J. Van Zwol, V. Svetovoy, and G. Palasantzas, Phys. Rev. B 80, 235401 (2009).

${ }^{25}$ See supplementary material at http://dx.doi.org/10.1063/1.4801459 for a description of the switchable friction experimental device and demonstration movie.

${ }^{26}$ Y. Mo, K. T. Turner, and I. Szlufarska, Nature 457, 1116 (2009).

${ }^{27}$ D. B. Asay and S. H. Kim, J. Phys. Chem. B 109, 16760 (2005).

${ }^{28}$ V. L. Popov, Contact Mechanics and Friction: Physical Principles and Applications (Springer, Berlin, 2010), Ch. 6 and 7.

${ }^{29}$ D. Liu, J. Martin, and N. Burnham, Appl. Phys. Lett. 91, 043107 (2007).

${ }^{30}$ H. R. Fischer and E. R. M. Gelinck, Appl. Surf. Sci. 258, 9011 (2012).

${ }^{31}$ J. Visser, Adv. Colloid Interface Sci. 3, 331 (1972). 